\title{
Angiotensin converting enzyme inhibitors and angiotensin receptor blockers improved the outcome of patients with severe COVID-19 and hypertension
}

\author{
Xiao Meng ${ }^{1,2}$, Ya Liu ${ }^{3}$, Chenchen Wei ${ }^{1}$, Kai Zhang ${ }^{1,2}$, Yi Zhang ${ }^{2,4}$, Ming Zhong ${ }^{1}$, \\ Cheng Zhang ${ }^{1 *} \&$ Yun Zhang ${ }^{1 *}$ \\ ${ }^{1}$ The Key Laboratory of Cardiovascular Remodeling and Function Research, Ministry of Education of China, Chinese National Health \\ Commission and Chinese Academy of Medical Sciences, The State and Shandong Province Joint Key Laboratory of Translational \\ Cardiovascular Medicine, Department of Cardiology, Qilu Hospital, Cheeloo College of Medicine, Shandong University, Jinan 250012, China; \\ ${ }^{2}$ Department of Infectious Disease, Renmin Hospital of Wuhan University, Wuhan 430060, China; \\ ${ }^{3}$ Department of Gastroenterology, Renmin Hospital of Wuhan University, Wuhan 430060, China; \\ ${ }^{4}$ Department of Respiratory Medicine, Qilu Hospital, Cheeloo College of Medicine, Shandong University, Jinan 250012, China
}

Received July 19, 2020; accepted September 1, 2020; published online September 21, 2020

Citation: Meng, X., Liu, Y., Wei, C., Zhang, K., Zhang, Y., Zhong, M., Zhang, C., and Zhang, Y. (2021). Angiotensin converting enzyme inhibitors and angiotensin receptor blockers improved the outcome of patients with severe COVID-19 and hypertension. Sci China Life Sci 64, 836-839. https://doi. $\operatorname{org} / 10.1007 / \mathrm{s} 11427-020-1813-0$

Dear Editor,

Hypertension was reportedly the most common coexisting condition of COVID- 19 as $15 \%-31.2 \%$ patients with COVID-19 had hypertension, and the incidence of hypertension reached 58.3\% in COVID-19 patients requiring ICU care (Wang et al., 2020). However, it remains unclear whether combined hypertension carries an increased risk for a worse outcome in patients with COVID-19 and what clinical factors independently predict death in these patients. Angiotensin converting enzyme inhibitors (ACEI) and angiotensin receptor blockers (ARB) are commonly used antihypertensive medications, but it is highly controversial whether ACEI/ARB treatment should be applied to patients with COVID-19 and hypertension because of a theoretical concern that these drugs may increase the expression of angiotensin converting enzyme 2 (ACE2), a high-affinity receptor of 2019-nCoV, thereby facilitating invasion of 2019-nCoV into the human body (Zhou et al., 2020).

*Corresponding authors (Yun Zhang, email: zhangyun@sdu.edu.cn; Cheng Zhang, email: zhangc@sdu.edu.cn)
To address these questions, a total of 580 patients admitted into Renmin Hospital of Wuhan University for COVID-19 from January 12, 2020 to March 27, 2020 were enrolled. Patients were divided into two groups according to their history of hypertension: hypertension (HT) group (blood pressure $\geq 140 / 90 \mathrm{mmHg}$ ) and normotension (NT) group (blood pressure $<140 / 90 \mathrm{mmHg}$ ). In addition, patients with hypertension were further divided into two subgroups: ACEI/ARB treatment (AT) sub-group and non-ACEI/ARB treatment (NAT) sub-group.

There were 300 men and 280 women, aged from 27 to 94 years and $\geq 60$ years in more than half patients. Patients in the HT group were older and had higher systolic and diastolic blood pressures (SBP, DBP) and more comorbidities such as stable coronary artery disease and diabetes, than those in the NT group. There were higher white blood cells, neutrophils, neutrophil-to-lymphocyte ratio (NLR) and serum levels of C-reactive protein (CRP), creatinine, blood glucose, triglycerides (TG), lactate dehydrogenase (LDH), and creatine kinase-myocardial isoenzyme (CK-MB) in the HT than the NT group. In contrast, the counts of platelet and 
CD8 cells were significantly lower in the HT than the NT group. Among 580 enrolled patients, 74 and 40 individuals in HT and NT groups, respectively, exhibited an increased cTnI level (28.6\% vs. $12.5 \%)$, suggesting that hypertension was associated with an increased risk of acute myocardial injury in patients with COVID-19. Patients in the HT group received more invasive mechanical ventilation than in the NT group $(8.5 \%$ vs. 3.1\%). In-hospital mortality was significantly higher in HT than in NT group $(21.2 \%$ vs. $10.3 \%$, $P<0.05$, Table $\mathrm{S} 1$ in Supporting Information).

In the subgroup analysis, among 259 patients in the HT group, 73 received $\mathrm{ACEI} / \mathrm{ARB}$ treatment (AT sub-group), while 186 received non-ACEI/ARB treatment (NAT subgroup). Patients in the AT sub-group showed lower neutrophil counts, NLR, serum levels of CRP, blood glucose and $\mathrm{LDH}$, and higher lymphocyte and platelet counts, than those in the NAT sub-group $(P<0.05)$. Importantly, 14 and 60 patients in the AT and NAT sub-group, respectively, exhibited elevated cTnI levels $(19.2 \%$ vs. $32.3 \%, P<0.05)$. Furthermore, patients in the NAT sub-group showed lower CD3, CD4 and CD8 cell counts than in the AT sub-group $(P<0.05)$. In the NAT sub-group, $51(27.4 \%)$ patients died while in the AT sub-group, only $4(5.5 \%)$ died. Noticeably, the differences in characteristics, laboratory findings and outcomes between the AT and NAT subgroups remained significant even after propensity score matching (Table 1). These results suggested that ACEI/ARB therapy may play a beneficial role in improving the clinical outcome in patients with severe COVID-19 and hypertension.

Multivariate regression analysis showed that dyspnea, increased age, serum levels of LDH and NLR, decreased CD3 cell counts and elevated serum levels of cTnI were significant and independent predictors of death in all COVID19 patients with and without hypertension (Table S2 in Supporting Information). In patients with combined COVID19 and hypertension, however, only decreased CD3 cell counts and elevated serum levels of cTnI were independent predictors of death. Noticeably, less use of ACEI/ARB was identified as a powerful and independent predictor of death in these patients, lending support to the notion that treatment with ACEI/ARB improves the outcome of patients with combined COVID-19 and hypertension (Table S3 in Supporting Information).

To analyze the effect of elevated blood pressure on clinical outcome in patients with combined COVID-19 and hypertension, we further divided the HT group patients into controlled blood pressure (blood pressure $<140 / 90 \mathrm{mmHg}$ on admission) and uncontrolled blood pressure (SBP $\geq 140 \mathrm{mmHg}$ and/or DBP $\geq 90 \mathrm{mmHg}$ on admission) subgroups. The results showed that patients in the uncontrolled blood pressure sub-group had an older age, higher SBP, DBP and serum levels of CK-MB and cTnI, and more use of diuretics than those in the controlled blood pressure sub- group. However, after propensity score matching, only SBP, DBP and serum levels of cTnI remained different between the two subgroups, indicating more patients with uncontrolled blood pressure had myocardial injury than those with controlled blood pressure (Table S4 in Supporting Information), a finding similar to a higher serum level of cTnI in the HT than the NT group.

In this study, patients in the HT group were different from those in the NT group in four aspects: older age, higher blood pressure, more intensive systemic inflammation as reflected by increased white blood cells, neutrophils, NLR and CRP, and more significant myocardial injury. The elderly is more subject to viral infection and multiple organ damage, and hypertension may induce endothelial dysfunction and left ventricular hypertrophy. It has been reported that viral replication may provoke apoptosis of respiratory epithelial and vascular endothelial cells, initiating a rapid production of proinflammatory cytokines and chemokines (Fu et al., 2020). Increased white blood cells, neutrophils and CRP were related to cytokine storm, which had been deemed as a predictor of a fatal outcome. The mildly increased serum level of cTnI in our patients is a definite indictor of myocardial injury, which has been recognized as a unique clinical feature of COVID-19 (Ruan et al., 2020). These differences between HT and NT groups may explain the higher in-hospital mortality and a lower discharge rate in HT than in NT group. Taken together, our results indicated that hypertension carries an increased risk for a worse outcome in patients with severe COVID-19.

Although respiratory failure is the leading cause of death in patients with COVID-19, myocardial injury has emerged as a secondary cause of death in these patients. In the current study, an elevated serum level of cTnI was detected in $28.6 \%$ and $12.5 \%$ patients in the HT and NT groups, respectively. The mechanism of an increased incidence of myocardial injury in the HT group is unclear but may be related to three potential mechanisms. First, the virus may directly invade myocardium leading to viral myocarditis, as ACE2, the receptor of 2019-nCoV, is widely distributed in the human heart and patients in the HT group are likely susceptible to COVID-19 due to an older age (Zhou et al., 2020). Second, 2019-nCoV downregulates ACE2 expression by binding ACE2 with resultant endocytosis, and activating disintegrin and metalloprotease 17 (ADAM17) to cleave membranebound ACE2, which may lead to an increased level of angiotensin II (Ang II) (Yang et al., 2020). A recent study reported that the serum levels of Ang II and CRP were dramatically increased in patients with COVID-19, which was linearly related to viral load and lung injury (Liu et al., 2020). As Ang II is a powerful pro-inflammatory factor, an elevated serum level of Ang II may predispose myocardium to an acute injury induced by cytokine storm. Third, hypoxemia as a consequence of respiratory failure may cause 
Table 1 Comparison of patients with combined COVID-19 and hypertension receiving ACEI/ARB and non-ACEI/ARB treatment before and after propensity score matching ${ }^{\text {a) }}$

\begin{tabular}{|c|c|c|c|c|c|c|}
\hline & \multicolumn{3}{|c|}{ Unmatched } & \multicolumn{3}{|c|}{ Matched (1:2) } \\
\hline & AT sub-group $(n=73)$ & NAT sub-group $(n=186)$ & $P$ values & AT sub-group $(n=73)$ & NAT sub-group $(n=133)$ & $P$ values \\
\hline Age (years) & $66.0(57.5-74.5)$ & $69.0(61.0-76.0)$ & 0.109 & $66.0(57.5-74.5)$ & $68.0(59.5-74.0)$ & 0.581 \\
\hline \multicolumn{7}{|l|}{ Sex } \\
\hline Male & $33(45.2 \%)$ & $105(56.5 \%)$ & \multirow{2}{*}{0.103} & $33(45.2 \%)$ & $66(49.6 \%)$ & \multirow{2}{*}{0.544} \\
\hline Female & $40(54.8 \%)$ & $81(43.5 \%)$ & & $40(54.8 \%)$ & $67(50.4 \%)$ & \\
\hline Heart rate (bpm) & $84.0(76.0-94.0)$ & $85.0(78.0-99.0)$ & 0.237 & $84.0(76.0-94.0)$ & $85.0(78.0-98.5)$ & 0.305 \\
\hline $\mathrm{SBP}(\mathrm{mmHg})$ & $135.0(121.5-146.0)$ & $136.0(121.8-150.3)$ & 0.859 & $135.0(121.5-146.0)$ & $136.0(123.0-150.5)$ & 0.718 \\
\hline DBP $(\mathrm{mmHg})$ & $79.0(70.5-87.5)$ & $78.0(71.0-89.3)$ & 0.769 & $79.0(70.5-87.5)$ & $78.0(70.0-87.5)$ & 0.591 \\
\hline White blood cells $\left(10^{9} \mathrm{~L}^{-1}\right)$ & $5.76(4.96-7.79)$ & $6.81(4.92-9.40)$ & 0.109 & $5.76(4.96-7.79)$ & $6.76(4.90-9.45)$ & 0.135 \\
\hline Neutrophils $\left(10^{9} \mathrm{~L}^{-1}\right)$ & $3.96(2.85-5.67)$ & $5.20(3.59-7.81)$ & 0.004 & $3.96(2.85-5.67)$ & $5.15(3.64-7.98)$ & 0.007 \\
\hline Lymphocytes $\left(10^{9} \mathrm{~L}^{-1}\right)$ & $1.14(0.83-1.68)$ & $0.85(0.60-1.23)$ & $<0.001$ & $1.14(0.83-1.68)$ & $0.85(0.61-1.32)$ & 0.001 \\
\hline NLR & $3.33(2.14-6.34)$ & $6.18(3.28-11.68)$ & $<0.001$ & $3.33(2.14-6.34)$ & $6.17(3.19-11.60)$ & $<0.001$ \\
\hline Hemoglobin $\left(\mathrm{g} \mathrm{L}^{-1}\right)$ & $123.0(113.5-131.5)$ & $123.0(112.5-136.0)$ & 0.746 & $123.0(113.5-131.5)$ & $122.0(111.0-134.5)$ & 0.997 \\
\hline Platelet $\left(10^{9} \mathrm{~L}^{-1}\right)$ & $226.0(182.5-280.0)$ & $201.0(150.8-270.5)$ & 0.023 & $226.0(182.5-280.0)$ & $200.0(152.5-260.0)$ & 0.025 \\
\hline $\mathrm{CRP}\left(\mathrm{mg} \mathrm{L}^{-1}\right)$ & $17.5(0-67.9)$ & $60.9(16.4-109.3)$ & $<0.001$ & $17.5(0-67.9)$ & $59.6(13.0-100.7)$ & 0.002 \\
\hline $\operatorname{ALT}\left(\mathrm{U} \mathrm{L}^{-1}\right)$ & $25.5(16.0-46.3)$ & $24.0(17.0-40.8)$ & 0.953 & $25.5(16.0-46.3)$ & $24.0(17.0-42.0)$ & 0.972 \\
\hline $\operatorname{AST}\left(\mathrm{U} \mathrm{L}^{-1}\right)$ & $25.0(18.0-38.8)$ & $30.0(20.0-47.0)$ & 0.052 & $25.0(18.0-38.8)$ & $30.0(20.0-47.0)$ & 0.117 \\
\hline Creatinine $\left(\mu \mathrm{mol} \mathrm{L}^{-1}\right)$ & $64.5(51.3-81.5)$ & $66.0(53.0-84.0)$ & 0.472 & $64.5(51.3-81.5)$ & $65.0(53.0-84.0)$ & 0.748 \\
\hline Blood glucose $\left(\mathrm{mmol} \mathrm{L}^{-1}\right)$ & $5.79(5.20-7.46)$ & $6.62(5.46-9.28)$ & 0.013 & $5.79(5.20-7.46)$ & $6.61(5.44-9.19)$ & 0.040 \\
\hline TG $\left(\mathrm{mmol} \mathrm{L}^{-1}\right)$ & $1.29(1.03-1.75)$ & $1.24(0.98-1.67)$ & 0.429 & $1.29(1.03-1.75)$ & $1.25(1.00-1.67)$ & 0.569 \\
\hline $\mathrm{TC}\left(\mathrm{mmol} \mathrm{L}^{-1}\right)$ & $4.06(3.36-4.44)$ & $3.64(3.13-4.23)$ & 0.063 & $4.06(3.36-4.44)$ & $3.70(3.30-4.28)$ & 0.168 \\
\hline LDL-C $\left(\mathrm{mmol} \mathrm{L}^{-1}\right)$ & $2.38(1.90-2.91)$ & $2.29(1.80-2.75)$ & 0.185 & $2.38(1.90-2.91)$ & $2.29(1.81-2.81)$ & 0.293 \\
\hline $\mathrm{LDH}\left(\mathrm{U} \mathrm{L}^{-1}\right)$ & $253.0(214.0-310.0)$ & $339.5(238.8-481.8)$ & $<0.001$ & $253.0(214.0-310.0)$ & $340.0(238.5-493.0)$ & 0.001 \\
\hline CK-MB (ng mL ${ }^{-1}$ ) & $1.27(0.89-1.95)$ & $1.45(0.89-2.97)$ & 0.071 & $1.27(0.89-1.95)$ & $1.38(0.88-2.96)$ & 0.141 \\
\hline Elevated cTnI & $14(19.2 \%)$ & $60(32.3 \%)$ & 0.036 & $14(19.2 \%)$ & $44(33.1 \%)$ & 0.034 \\
\hline $\operatorname{CD} 3\left(\mu \mathrm{L}^{-1}\right)$ & $639.0(437.0-1009.0)$ & $505.0(306.0-767.0)$ & 0.002 & $639.0(437.0-1009.0)$ & $516.0(306.0-780.0)$ & 0.010 \\
\hline $\mathrm{CD} 4\left(\mu \mathrm{L}^{-1}\right)$ & $422.0(265.0-606.0)$ & $320.0(183.0-488.0)$ & 0.001 & $422.0(265.0-606.0)$ & $320.0(172.0-505.0)$ & 0.006 \\
\hline $\operatorname{CD} 8\left(\mu \mathrm{L}^{-1}\right)$ & $212.0(116.0-326.0)$ & $163.0(90.0-260.0)$ & 0.011 & $212.0(116.0-326.0)$ & $165.0(95.0-272.0)$ & 0.031 \\
\hline \multicolumn{7}{|l|}{ Antihypertensive treatment } \\
\hline$\beta$-blocker & $24(32.9 \%)$ & $37(19.9 \%)$ & 0.027 & $24(32.9 \%)$ & $27(20.3 \%)$ & 0.045 \\
\hline $\mathrm{CCB}$ & $48(65.8 \%)$ & $107(57.5 \%)$ & 0.224 & $48(65.8 \%)$ & $73(54.9 \%)$ & 0.130 \\
\hline Diuretic & $17(23.3 \%)$ & $40(21.5 \%)$ & 0.755 & $17(23.3 \%)$ & $25(18.8 \%)$ & 0.444 \\
\hline \multicolumn{7}{|l|}{ Clinical outcome } \\
\hline Discharge & $69(94.5 \%)$ & $135(72.6 \%)$ & \multirow{2}{*}{$<0.001$} & $69(94.5 \%)$ & $98(73.7 \%)$ & \multirow{2}{*}{$<0.001$} \\
\hline Death & $4(5.5 \%)$ & $51(27.4 \%)$ & & $4(5.5 \%)$ & $35(26.3 \%)$ & \\
\hline
\end{tabular}

a) Data were expressed as $n(\%)$ and median (IQR). ACEI: angiotensin converting enzyme inhibitors; ARB: angiotensin receptor blockers; SBP: systolic blood pressure; DBP: diastolic blood pressure; NLR: neutrophil-to-lymphocyte ratio; CRP: C-reactive protein; ALT: alanine aminotransferase; AST: aspartate aminotransferase; TG: triglyceride; TC: total cholesterol; LDL-C: low density lipoprotein cholesterol; LDH: lactate dehydrogenase; CK-MB: creatine kinasemyocardial isoenzyme; cTnI: cardiac troponin I; CCB: calcium channel blockers.

myocardial ischemia, thus aggravating coronary microvessel disease commonly seen in HT patients.

Since human ACE2 is recognized as the cell binding receptor of 2019-nCoV (Zhou et al., 2020), the relation between ACE2 and ACEI/ARB treatment has received increasing attention. Fang et al. proposed that ACEI/ARB treatment may increase ACE2 expression, which may facilitate COVID-19 infection and multiply the risk for severe COVID-19 (Fang et al., 2020). This speculation aroused serious concerns about the safety of ACEI/ARB in patients with COVID-19 and hypertension. However, the effect of ACEI/ARB on the expression and activity of ACE2 has 
never been confirmed in either experimental research or clinical practice. In a rat model of myocardial infarction, Burchill et al. reported that neither ramipril or valsartan alone nor in combination enhanced cardiac ACE2 expression, suggesting their cardioprotective effects were not mediated through up-regulation of cardiac ACE2 (Burchill et al., 2012). Our results demonstrated that patients in the AT sub-group had a lower level of systemic inflammation and myocardial injury than the NAT group, which was consistent with the RAICES study in which serum levels of CRP were significantly reduced after a 6-month ramipril therapy in 77 patients with coronary artery disease (Lopez Santi et al., 2005). The mechanism underlying the benefit of ACEI/ARB therapy in our patients is unknown but may be mainly due to the inhibition of Ang II production by ACEI or the blockade of Ang II receptor type 1 (AT1R) by ARB. It has been reported that Ang II may activate ADAM-17 to cleave ACE2 off cell membrane and ACEI/ARB therapy may restore ACE2-angioten-(1-7)-Mas axis to antagonize overactive renin-angiotensin system induced by COVID-19 (Jiang et al., 2014). In addition, CD3, CD4 and CD8 T cell counts were higher in AT than NAT group. Lymphocytopenia is commonly seen in patients with COVID-19, which may indicate an impaired immune response (Chen et al., 2020; Fu et al., 2020), and ACEI/ARB treatment restored normal immune function in these patients. Eventually, these salutary effects of ACEI/ARB therapy translated into a lower in-hospital mortality and a higher discharge rate in the AT than the NAT group. Our results provided direct evidence that ACEI/ARB treatment is beneficial for improving clinical outcomes of patients with severe COVID19 and hypertension.

In conclusion, we demonstrated that patients with combined COVID-19 and hypertension had more significant systemic inflammation and acute myocardial injury, a higher in-hospital mortality and a lower discharge rate, whereas COVID-19 and hypertensive patients receiving ACEI/ARB therapy had an improved clinical outcome. Finally, an elevated serum level of cTnI and less use of ACEI/ARB therapy were strong and independent predictors of death in patients with combined COVID-19 and hypertension. Further randomized, multicenter and controlled studies are warranted to confirm these preliminary findings.
Compliance and ethics The author(s) declare that they have no conflict of interest.

Acknowledgements We thank all patients involved in this study. This study was supported by the Program of Introducing Talents of Discipline to Universities (BP 0719033), the State Key Program of the National Natural Science Foundation of China (81530014) and the International Collaboration and Exchange Program of China (81920108003).

\section{References}

Burchill, L.J., Velkoska, E., Dean, R.G., Griggs, K., Patel, S.K., and Burrell, L.M. (2012). Combination renin-angiotensin system blockade and angiotensin-converting enzyme 2 in experimental myocardial infarction: implications for future therapeutic directions. Clin Sci $123,649-658$.

Chen, N., Zhou, M., Dong, X., Qu, J., Gong, F., Han, Y., Qiu, Y., Wang, J., Liu, Y., Wei, Y., et al. (2020). Epidemiological and clinical characteristics of 99 cases of 2019 novel coronavirus pneumonia in Wuhan, China: a descriptive study. Lancet 395, 507-513.

Fang, L., Karakiulakis, G., and Roth, M. (2020). Are patients with hypertension and diabetes mellitus at increased risk for COVID-19 infection? Lancet Respir Med 8, e21.

$\mathrm{Fu}$, Y., Cheng, Y., and Wu, Y. (2020). Understanding SARS-CoV-2mediated inflammatory responses: from mechanisms to potential therapeutic tools. Virol Sin 35, 266-271.

Jiang, F., Yang, J., Zhang, Y., Dong, M., Wang, S., Zhang, Q., Liu, F.F., Zhang, K., and Zhang, C. (2014). Angiotensin-converting enzyme 2 and angiotensin 1-7: novel therapeutic targets. Nat Rev Cardiol 11, 413426.

Liu, Y., Yang, Y., Zhang, C., Huang, F., Wang, F., Yuan, J., Wang, Z., Li, J., Li, J., Feng, C., et al. (2020). Clinical and biochemical indexes from 2019-nCoV infected patients linked to viral loads and lung injury. Sci China Life Sci 63, 364-374.

Lopez Santi, R.G., Valeff, E.C., Duymovich, C.R., Mazziotta, D., Mijailovsky, N.E., Filippa, G.C., Maltez, R., Hernandez, V.A., Monroy, A.G., Borzi, J.G., et al. (2005). Effects of an angiotensinconverting enzyme inhibitor (ramipril) on inflammatory markers in secondary prevention patients: RAICES Study. Coronary Artery Dis 16, 423-429.

Ruan, Q., Yang, K., Wang, W., Jiang, L., and Song, J. (2020). Clinical predictors of mortality due to COVID-19 based on an analysis of data of 150 patients from Wuhan, China. Intensive Care Med 46, 846-848.

Wang, D., Hu, B., Hu, C., Zhu, F., Liu, X., Zhang, J., Wang, B., Xiang, H., Cheng, Z., Xiong, Y., et al. (2020). Clinical characteristics of 138 hospitalized patients with 2019 novel coronavirus-infected pneumonia in Wuhan, China. JAMA 323, 1061-1069.

Yang, J.M., Meng, X., Xue, F., Zhang, Y., and Zhang, C. (2020). ACE2 in the context of 2019-nCoV infection: friend or foe? Zhonghua Xin Xue Guan Bing Za Zhi 48, 527-531.

Zhou, P., Yang, X.L., Wang, X.G., Hu, B., Zhang, L., Zhang, W., Si, H.R., Zhu, Y., Li, B., Huang, C.L., et al. (2020). A pneumonia outbreak associated with a new coronavirus of probable bat origin. Nature 579, $270-273$.

\section{SUPPORTING INFORMATION}

The supporting information is available online at https://oi.org/10.1007/s11427-020-1813-0. The supporting materials are published as submitted, without typesetting or editing. The responsibility for scientific accuracy and content remains entirely with the authors. 